\title{
A Novel Fault Diagnosis System for Blast Furnace Based on Support Vector Machine Ensemble
}

\author{
Huixin $\operatorname{TIAN}^{1)}$ and Anna WANG ${ }^{21}$ \\ 1) School of Electrical and Automation Engineering, Tianjin Polytechnic University, Tianjin, 300160 P. R. China. \\ E-mail: icedew|@163.com 2) Information Science and Engineering Department, Northeastern University, Shenyang, \\ 110004 P. R. China.
}

(Received on October 28, 2009; accepted on March 4, 2010)

\begin{abstract}
Fault diagnosis plays an important role during the process of blast furnace ironmaking for producing safety. In this paper the important parameters of puddling process are selected as judgments criterions of fault diagnosis by analyzing the changes of these parameters. Support vector machine (SVM) is used to establish the fault diagnosis model for its suitable characters for fault classification. But the stability and accuracy of model based on single SVM could not meet the needs of practical ironmaking. Therefore, a SVM ensemble based on bagging is presented to establish a novel fault diagnosis system. The real-time producing data are collected in 5\# blast furnace of a steel enterprise for training and testing the fault diagnosis models with single SVM and SVM ensemble. The experiments about the comparison between single SVM and SVM ensemble and about the SVM ensembles with different number of individual SVM are made. The experimental results demonstrate that the performance of novel fault diagnosis system based on SVM ensemble is better than the one based on single SVM, and the best fault diagnosis system that can meet the practical needs of ironmaking is found.
\end{abstract}

KEY WORDS: blast furnace; support vector machine; ensemble technique; bagging.

\section{Introduction}

Blast furnace is a principal industrial unit for production of molten iron for primary steelmaking in modern iron and steel industry. It is a mass producer of hot metal for the following steel manufacturing processes. Therefore, in a relatively long period, the blast furnace still maintains its predominant position.

During the whole industry process of blast furnace ironmaking, there are a lot of parameters from adding material, blasting, and adding fuel to the iron bearing materials (ores) being reduced into hot iron. ${ }^{1)}$ These parameters could reflect the states of producing and equipments in blast furnace ironmaking. Therefore, it is necessary to measure these parameters and observe or analyze the changes of these parameters. According to the conclusions of above analyzing and estimating, we could determine whether the produce and equipments are in normal states or fault states. Meanwhile, the fault will be found at the first time, and will be removed in the shortest time for avoiding the great losses efficiently. In a word, it is great significant to establish a good fault diagnosis system of blast furnace based on the process parameters to ensure the following metallurgic producing and enhance the economic benefit.

The fault diagnosis problem could be formulated as a kind of fault classification. Recently, with the fast development of intelligent technique, more and more intelligent methods have been used in real industrial production widely. Support vector machine (SVM) is a kind of method being suitable for the fault diagnosis. SVM has a good generalization capability even in the small sample cases of classification. ${ }^{2)}$ In recent years, SVM has been successfully applied for solving the classification problem, but there are still some problems in the practical application of SVM. The one is how to select kernel function and SVM parameters (the parameters of kernel function and regularization constant). An unsuccessful selection will make the performance of SVM unstable. Furthermore, the accuracy of single SVM does not satisfy some practical applications.

In this paper the SVM will be used to establish the fault diagnosis models for the $5 \#$ blast furnace of a steel enterprise. For the sake of enhancing the stability and the accuracy of the fault diagnosis system with single SVM, a SVM ensemble scheme is used to aggregate the single SVM models and a novel fault diagnosis model is established for blast furnace. The real production data of the $5 \#$ blast furnace from 2006 to 2007 is used to build the fault diagnosis system based on SVM ensemble and test the performance of fault diagnosis system. The experiments demonstrate that the novel fault diagnosis system can diagnose the fault efficiently and the performance of fault diagnosis model based on SVM ensemble is better than the one based on SVM.

\section{Blast Furnace and Important Process Parameters}

The blast furnace is a counter current chemical reactor whose main purpose is to reduce iron oxides to iron. A typ- 
ically furnace is $20-30 \mathrm{~m}$ high and has a diameter of $10 \mathrm{~m}$. The heat and mass transfer as well as chemical reactions will occur between several phases. The raw materials, i.e., iron bearing materials (ores) and the main fuel, metallurgical coke, are charged layer by layer at the top of the furnace shaft. In the lower part of the furnace, preheated air, blast, and additives, such as oil or pulverized coal, humidity and oxygen are injected through tuyeres. The burning of coke in front of the tuyeres makes the bed of burden descend. The combustion gas consists of mainly carbon monoxide (CO) and nitrogen $\left(\mathrm{N}_{2}\right)$. It rises through the burden and heats, reduces, and melts simultaneously the ores. The pressure of combustion gas may vary between 5 and 1 bar (depending on the size of the furnaces). The combustion gas in a partially oxidized state leaves the furnace top together with approximately equal parts of carbon monoxide and carbon dioxide. The molten pig iron and slag collect on the bottom or hearth of the furnace in turn and are tapped more or less regularly through the taphole via runners to torpedo cars or ladles, which are sent downstream for steel processing. In modern furnaces using large scale injection of pulverised coal (PC) or other particulate materials, fine solids should also be considered a major phase. The furnace temperature varies from a few hundred degrees (upper shaft) to more than 2000 degrees in the combustion zone. The molten pig iron is tapped at about $1450-1550^{\circ} \mathrm{C}$. It consists of mainly iron $(\approx 94 \% \mathrm{Fe})$, dissolved carbon $(\approx 4-5 \%)$, silicon $(\approx 0.2-1.0 \%)$ and with smaller amounts of alloying elements (such as manganese, phosphorus, titanium, vanadium, etc.). It is very difficult to measure the inner state of ironmaking process directly for the high temperatures, pressure, and the mechanical wear. The average residence time of the solid/liquid phase is $5-10 \mathrm{~h}$, while the gas passes through the process in 10-20 s. ${ }^{3,4)}$

According to the above ironmaking process of blast furnace, several important parameters are selected as the criterions of fault diagnosis. They are the temperature $\left({ }^{\circ} \mathrm{C}\right)$ of top of furnace, furnace pressure $(\mathrm{kPa})$, material addition velocity $($ batch/h), venting quality, pressure difference $(\mathrm{kPa})$, blast volume $\left(\mathrm{m}^{3} / \mathrm{min}\right)$, blast pressure $(\mathrm{Pa})$, blast temperature $\left({ }^{\circ} \mathrm{C}\right)$, and position of material $(\mathrm{m})$. In the real production process of blast furnace, the temperatures are measured by thermocouples, the pressure is measured by pressure sensor, and the venting quality is calculated by blast volume and blast pressure. The fault will be diagnosed by the analysis of the change of above parameters. These parameters are used as the inputs of the fault diagnosis system of blast furnace to detect whether the blast furnace system runs in a normal state.

\section{Support Vector Machine}

Support vector machine (SVM) was originally introduced by Vapnik et al. 1999. Now SVM has become an increasingly popular technique for machine learning activities including classification, regression, outlier detection etc. It obtains real risk minimization by seeking for structural risk minimization. SVM integrates the optimal separating hyperplane with the kernel method. It could overcomes commendably such defects as dimensionality curse and overfitting that are apt to appear in some other conventional algo- rithms, neural net for instance. The idea of using SVM for separating two classes is to find support vectors to define the bounding planes, in which the margin between the both planes is maximized. The number of support vectors increases with the complexity of the problem. ${ }^{5-7)}$

To define SVM mathematically, the training data are first stacked into a $n \times m$ matrix $X$, where $n$ is the number of observations and $m$ is the number of variables. Denote $x_{i}$ as a column vector representing the $i$ th row of $X$. A $n \times n$ diagonal matrix $Y$ with +1 and -1 entries is then used to specify the membership of each $x_{i}$ in class +1 or -1 . Observations in both classes satisfy.

$$
Y(X w-e \gamma) \geq e .
$$

where $e$ is a vector of ones. To determine $w$ and $\gamma$, the following mathematical program is solved

$$
\min \frac{1}{2}\|w\|^{2} \text { s.t. } Y(X w-e \gamma) \geq e
$$

To determine $w$ and $\gamma$, quadratic programming (QP) can be solved by finding the saddle point of the Lagrange functional (Scholkopf and Smola, 2002). The observations for which the corresponding Lagrange multipliers are positive are referred to as support vectors. With $w$ and $\gamma$ being solved, the class membership of $x$ is determined as

$$
y_{\text {pred }}=\operatorname{sign}\left(w^{T} x+\gamma\right) \ldots
$$

For data that overlap, nonnegative slack variable $\xi$ is introduced into the mathematical program

$$
\min \frac{1}{2}\|w\|^{2}+C\|\xi\| \text { s.t. } Y(X w-e \gamma)+\xi \geq e, \xi \geq 0
$$

The first term in the objective function represents the model complexity and the second term represents the model accuracy (i.e. classification error in the training data). The regularization parameter $C$ controls the trade-off between these two terms. Model underfitting occurs when $C$ is too small.

The merit of support vector machine is that, by a kernel function, which is the inner product in the feature space, it ties to make the training data linear-separable in the high dimension feature space, thus achieve nonlinear-separable in the input space. Typical choices of kernel function include the follows:

Linear kernel function

$$
K\left(x_{i}, y_{j}\right)=x_{i} \cdot y_{j}
$$

Polynomial kernel function:

$$
K\left(x_{i}, y_{j}\right)=\left[\left(x_{i} \cdot y_{j}\right)+r\right]^{d} \text {. }
$$

Radial-basis function (RBF) kernel (also named as Gaussian kernel)

$$
K\left(x_{i}, y_{j}\right)=\exp \left(-\lambda\left|x_{i}-y_{j}\right|^{2}\right)
$$

Sigmoid kernel function:

$$
K\left(x_{i}, y_{j}\right)=\tanh \left(v\left(x_{i}^{T} \cdot y_{j}\right)-\delta\right)
$$

where $d$ is the exponent of polynomial function, $r, \lambda, v$ and $\delta$ are kernel parameters.

When the SVM is used in practical industrial application, 
the users have to face the problem that how to select a suitable kernel function and SVM parameters to ensure a good performance of SVM. In the flowing section a new SVM ensemble approach will be proposed to overcome the above problem.

\section{SVM Ensemble}

Ensemble technique has become an increasingly popular technique for its great capability of enhancing the performance of individual machine. It aggregates the multiple classifiers to produce a single classifier in some way. The ensemble of machines is often called a committee machine. In a committee machine, an ensemble of classifiers is generated by means of a learning process; the overall classifiers of the committee machine are the combination of the individual committee members' classifiers. And various ensemble techniques have been investigated by many researchers. Several studies of ensemble techniques (such as boosting and bagging) in classification have demonstrated that these techniques are generally more accurate than the individual classifiers. To obtain a good ensemble, two main problems should be solved: how to construct accurate and diverse base classifiers and how to combine their outputs effectively. Consequently, various ensemble techniques are different mainly in two ways: construct scheme and combining scheme. $^{8-10)}$

\subsection{Construct Scheme}

Many methods for constructing ensembles have been developed. The most prominent ensemble machine generation methods are Bagging (Breiman, 1996) and Boosting (Freund and Schapir, 1996). Bagging employs bootstrap sampling to generate different training sets that consists of $N$ train examples drawn randomly with replacement from the original training set of $N$ items. Such training set is called bootstrap replicate. Bagging trains a classifier on each training set to build its constituent members, which can be generated in parallel.

Boosting works by repeatedly running a given weak learning machine on different distribution of training data and combining their outputs. In each iteration, the distributions of training examples depend on the performance of the machine in the previous iteration. The main idea of boosting is to train a sequence of classifier with the socalled boosting sets so that each subsequent classifier concentrates mostly on the errors made by the previous ones. This is achieved by maintaining a set of weights over the whole training examples.

\subsection{Combining Scheme}

After constructing a good ensemble of classifiers, it is also necessary manipulate the output values that are given to the learning algorithm. A variety of combining scheme have been developed and used for classifier ensemble to manipulate the output in the literatures. The most often used and popular schemes are the average, weight and majority vote combining. Average approach averages the individual classifier outputs across all the members. Weight approach combines the outputs according to the performance of individual classifier. That is to say, the better classifier will be given a big value of weight, otherwise will be given a small value of weight. In majority vote approach the output will be controlled by the classifiers that have majority votes. It is the most popular aggregation method for its easy implementation.

\subsection{Development of the Model Based on SVM Ensem- ble}

In order to improve the performance of fault diagnosis system, the SVM ensemble is used to establish the fault diagnosis model. Firstly, the individual SVMs are constructed with different kernel function and different kernel parameters. The kernel functions involve Polynomial kernel, RBF kernel and Sigmoid kernel. The kernel parameters and regularization constant $C$ are chosen randomly in their valid range. Thus, different Polynomial kernel SVM classifiers, RBF kernel SVM classifiers and Sigmoid kernel SVM classifiers are established by this approach. Secondly, the SVM ensemble is established using bagging ensemble technique. The data sub-sets with the same number of data are obtained by bootstrap method from original data set. The individual SVMs are chosen randomly in above generated SVM classifiers as sub-learning machine of Bagging. Every individual SVM is trained by corresponding data sub-set. At the end, these individual SVMs can be aggregated by majority vote combining scheme as Eq. (9). So a fault diagnosis model based on SVM ensemble is established.

$$
h_{\text {fin }}(x)=\arg \max _{c} \sum_{t: h_{t}(x)=c} 1
$$

Where, $h_{i}=$ classifier $\left(S_{i}\right), S_{i}$ are data sub-sets, $i=1,2, \ldots, T$ is the numbers of sub-learning machine.

\section{Experiments}

In this section the fault diagnosis system for blast furnace based on SVM ensemble will be presented by using matlab. The matlab will also be used for individual SVM training and classification. Ten kinds of state including normal state and nine kinds of abnormal state such as pipe fracture, cooler, warmer, slip, low stockline, brim gas flow, centre gas flow, hanging, moving handicap will be diagnosed. Nine process parameters (mentioned in Sec. 2) are selected to diagnose these states. The comparisons between the fault diagnosis models based on SVM ensemble and individual SVM will be carried out. In the end, the experiments about the SVM ensemble with different numbers of individual SVM will be done. The results of these experiments are used to discuss how the size of SVM ensemble affects the fault diagnosis of blast furnace.

\subsection{Data}

For establishing the fault diagnosis system for real producing process of blast furnace, the 578 real-time producing data are collected during the producing process of $5 \#$ blast furnace in a steel enterprise from October 2006 to August 2007. They involve nine process parameters and ten kinds of states (above mentioned). The 578 data are divided into two data sets; the one containing 300 data is used as training data set, and the other containing 278 data is used as test data set. 


\subsection{Individual SVM Classifiers with Different Kernel Function}

The Polynomial kernel, the RBF kernel and the Sigmoid kernel are used respectively in individual SVM classifiers. Every kind of individual SVM classifiers is trained 15 times by the training data set. In these individual SVM classifiers, the kernel parameters (involve $r, \lambda, v$ and $\delta$ ) and regularization constant $C$ are chosen randomly. These 45 individual SVM classifiers are also test by the same testing data set.

The experimental results of above individual SVM classifiers are showed in the Table 1. For each kind of individual SVM classifier (kernel function), the performance of fault diagnosis is reported by training accuracy and testing accuracy. Owning to there are 15 SVM classifiers with each kernel function, not only the average of accuracy is presented, but also the best and the worst of accuracy is presented.

\subsection{Comparison between SVM Ensemble and Individ- ual SVM Classifiers}

In this section, the SVM ensemble model based on bagging is established. The individual SVMs are chosen randomly from Sec. 5.2 as sub-learning machines of Bagging. The number of individual SVM sub-learning machines is 10. The fault diagnosis model based on the SVM ensemble is also trained 15 times with random sub-learning machine, i.e. random kernel function, random kernel parameters and random regularization constant $C$. The performance of fault diagnosis model based on SVM ensemble is displayed in Table 2. The average, best and worst of accuracy of SVM ensemble is also presented. The experimental results demonstrate the performance of SVM ensemble model is better than the individual SVM. Moreover, the SVM ensemble models yield stable results comparing to the individual SVM classifiers.

\subsection{Comparison of SVM Ensemble Classifiers with Different Numbers of Sub-learning Machine}

Although the bagging ensemble technique has been used

Table 1. The performance of fault diagnosing based on individual SVM classifiers with different kernel function.

\begin{tabular}{cccc}
\hline Kernel function & & Training accuracy (\%) & Testing accuracy (\%) \\
\hline Polynomial kernel & Average & 96.18 & 96.05 \\
& Best & 96.40 & 96.26 \\
& Worst & 95.93 & 95.82 \\
\multirow{5}{*}{ RBF kernel } & Average & 93.87 & 93.43 \\
& Best & 93.99 & 93.77 \\
& Worst & 93.67 & 93.15 \\
Sigmoid kernel & Average & 90.65 & 90.02 \\
& Best & 91.45 & 90.37 \\
& Worst & 90.00 & 89.73 \\
\hline
\end{tabular}

Table 2. The performance of fault diagnosing based on SVM ensemble.

\begin{tabular}{ccc}
\hline & Training accuracy (\%) & Testing accuracy (\%) \\
\hline Average & 96.65 & 96.34 \\
Best & 96.76 & 96.39 \\
Worst & 96.53 & 96.28 \\
\hline
\end{tabular}

in a wide range, in the former studies, there is no rule as to how many numbers of sub-learning machines should ensure a good performance of bagging. Obviously, it is beneficial to keep the number small for saving the training time. When the number of individual SVM classifier is increasing, the better characters can be obtained by combining schemes. Moreover, the complexity of the whole system is implicitly exposed too for the same reasons. It also should bring the over-fitting problem. How to design an ensemble model with an appropriate number of sub-learning machines is a necessary problem to be resolved. In this section, the experiment is done to study the behavior of the SVM ensemble models when the number of sub-learning machine increases.

The SVM ensemble models with different number of sub-learning machine are trained and test by above training data set and test data set. The number increases from 2 to 15. The corresponding results of experiments are showed in Fig. 1 and Fig. 2. Figure 1 shows the change of performance of training of SVM ensemble models; and Fig. 2 shows the change of performance of test of SVM ensemble models.

The figures indicated that the performance of SVM ensemble will become better when the number of individual SVM classifiers increases. When the ensembles are generated with 8-12 individual SVM classifiers, they can obtain relatively better performance. Beyond this number, the performance significant improves no longer, and even deteriorates in some cases. Therefore, we can draw a conclusion that the performance of SVM ensemble models based on

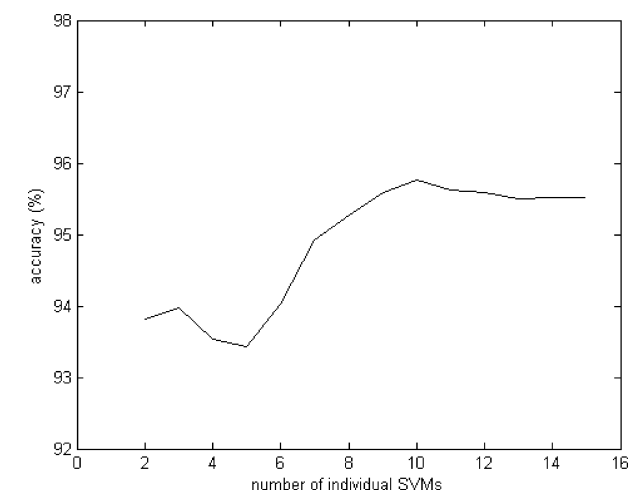

Fig. 1. Performance of SVM ensemble with different number of individual SVMs by training data.

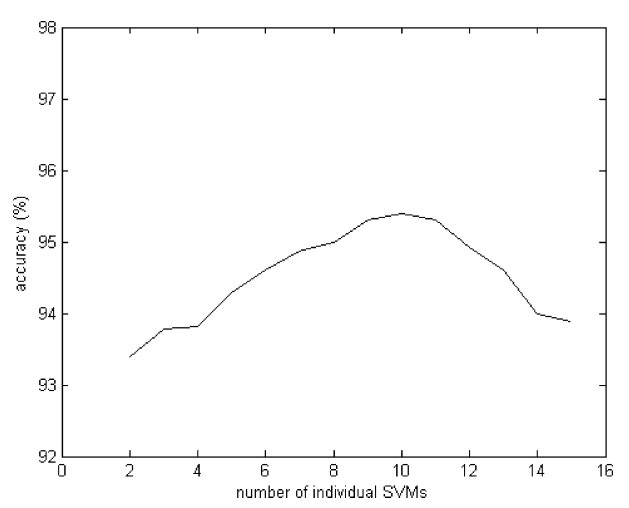

Fig. 2. Performance of SVM ensemble with different number of individual SVMs by test data. 
Table 3. The results of diagnosing by fault diagnosis system based on SVM ensemble.

\begin{tabular}{ccc}
\hline Fault states & Distortion rate (\%) & Omission rate (\%) \\
\hline pipe fracture & 0.3264 & 0 \\
cooler & 0.2514 & 0.1406 \\
warmer & 0.2015 & 0.1406 \\
slip & 0.3518 & 0.3021 \\
low stockline & 0 & 0 \\
brim gas flow & 0.1120 & 0 \\
centre gas flow & 0.2913 & 0.1406 \\
hanging & 0.2525 & 0.2842 \\
moving handicap & 0 & 0 \\
normal & 0 & 0 \\
\hline
\end{tabular}

bagging ensemble technique will not improve continuously according to the increase of the number of individual SVM classifiers. Furthermore, the experiments in the tests indicate that the ensemble SVMs may suffer from overfitting in the presence of larger number that can be illustrated by some of the decreases in performance.

Then a fault diagnosis system for blast furnace is established by the novel SVM ensemble method with 10 individual SVM sub-learning machines. Table 3 shows the results of diagnosis by this fault diagnosis system based on SVM ensemble. The diagnosis on normal and abnormal state could be identified correctly.

\section{Conclusion}

Fault diagnosis for blast furnace is necessary during the process of ironmaking for modern iron and steel industry. In this paper, the important process parameters of blast furnace are determined according to the practical puddling process of blast furnace. The fault diagnosis system would be established by using and analyzing these parameters. SVM is selected as the basic intelligent method to establish the fault diagnosis system for its better performance when it is used to solve the fault classification problem. Furthermore, aiming at the shortcoming of using single SVM, a SVM ensemble technique based on bagging method is presented to establish a novel fault diagnosis system. The realtime producing data are collected to train and test the fault diagnosis system in $5 \#$ blast furnace of a steel enterprise. The experiments between the model using single SVM and SVM ensemble and the experiments of SVM ensemble with different number of individual SVMs are made. The experimental results demonstrate that the performance of the fault diagnosis system based on SVM ensemble is better than the one based on single SVM, and that the SVM ensembles cannot continue to improve with larger number of individual SVMs. At the end, a best SVM ensemble model for fault diagnosis is found by experiments. The novel fault diagnosis system based on SVM ensemble can satisfy the needs of practical production.

\section{Acknowledgment}

This research is supported by National Natural Science Foundation of China (Grant No. 60843007).

\section{REFERENCES}

1) R. Ostermark and H. Saxen: Eur. J. Oper. Res., 90 (1996), 85.

2) C. J. C. Burges: Data Min. Knowl. Discov., 2 (1998), 121.

3) J. C. Song: Theory and Operation in Blast Furnace Puddling, Metallurgical Industry Press, Beijing, (2005), 179.

4) A. K. Biswas: Principles of Blast Furnace Ironmaking, Cootha Publishing House, Brisbane, (1981), 27.

5) E. M. Jordaan and G. F. Smits: Proc. of World Conf. on Computational Intelligence, IEEE, Honoluu Hawaii, (2002), 2785.

6) L. H. Chiang, M. E. Kotanchek and A. K. Kordon: Comput. Chem. Eng., 28 (2004), 1389.

7) S. Chen, W. Wang and H. van Zuylen: Expert Systems with Applications, 36 (2009), 10976.

8) I. Maqsood, M. R. Khan and Abraham: Neural Comput. Appl., 13 (2004), 112.

9) J. Demsar: J. Mach. Learn. Res., 7 (2006), 1.

10) E. Bauer and R. Kohavi: Mach. Learn., 24 (1998), 1. 